\title{
Absolute Blood Volume in Hemodialysis Patients: Why Is It Relevant, and How to Measure It?
}

\author{
Stephan Thijssen ${ }^{a}$ Franz Kappel $^{a, b}$ Peter Kotanko ${ }^{a}$ \\ ${ }^{a}$ Renal Research Institute, New York, N.Y., USA; ${ }^{b}$ Institute for Mathematics and Scientific Computing, University of \\ Graz, Graz, Austria
}

\section{Key Words}

Absolute blood volume Relative blood volume . Intradialytic hypotension $\cdot$ Crit-Line $\cdot \mathrm{F}$ cell ratio $\cdot$ Dry weight

\begin{abstract}
Intradialytic hypotension (IDH) is the most common complication during hemodialysis and is associated with significant morbidity. It occurs as a consequence of a reduction in blood volume during ultrafiltration. Today, devices for monitoring relative blood volume (i.e. the intradialytic blood volume as a fraction of the blood volume at the start of the dialysis treatment) are routinely available and have been used to analyze the relationship between changes in relative blood volume and the occurrence of IDH in an attempt to derive critical thresholds that, when observed, would help avoid hypotensive episodes during the treatment. However, the results have been unsatisfactory in many patients. Here we illustrate the importance of absolute blood volume in hemodialysis patients, summarize the acute physiologic mechanisms involved in the preservation of adequate circulation during ultrafiltration, and outline why hemodialysis patients are often particularly vulnerable to reductions in blood volume. We then discuss the shortcomings of relative blood volume measurements, make a case for the superiority of abso-
\end{abstract}

lute blood volume measurements, and introduce the reader to a mathematical concept that allows relative blood volume devices to be used for the estimation of absolute blood volume. Finally, we discuss the implications of absolute blood volume beyond IDH and propose a paradigm shift in the approach to dry weight attainment.

Copyright $\odot 2013$ S. Karger AG, Base

\section{Why Is Blood Volume of Importance in Hemodialysis Patients?}

In the United States, there were close to 400,000 prevalent hemodialysis patients in 2010 [1]. At a rate of three treatments per week, this adds up to about 62 million hemodialysis treatments per year. Intradialytic hypotension (IDH) is still the most frequent complication during the dialysis treatment with a frequency of about $15-30 \%$ (and perhaps higher) of all maintenance hemodialysis sessions [2, 3], for an estimated total of somewhere between 9 and 19 million IDH episodes in the United States in 2010. This is a substantial number and is largely a resultant of current hemodialysis practice, i.e. relatively short (3-4 h), infrequent ( 3 times per week) treatments during which the entire interdialytic fluid accumulation has to be corrected. Other aspects (such as intradialytic

\section{KARGER}

Fax +4161306 1234

E-Mail karger@karger.ch

www.karger.com
(C) 2013 S. Karger AG, Basel

0253-5068/13/0353-0063\$38.00/0

Accessible online at:

www.karger.com/bpu
Stephan Thijssen, MD

Research Laboratory Director, Renal Research Institute

207 East 94th Street, Suite 303

New York, NY 10128 (USA)

E-Mail sthijssen@rriny.com 
sodium loading via inappropriately high dialysate sodium concentrations or inappropriate use of sodium profiling, inadequate dietary counseling and priming/rinsing of the dialysis circuit with saline solution) may lead to increased interdialytic weight gains and, thereby, compound the problem [4].

This high rate of IDH is of concern because IDH is associated with several problems, among them mesenteric hypoperfusion with subsequent translocation of endotoxin into the bloodstream, cerebral damage, accelerated loss of residual renal function and cardiac damage $[5,6]$. Further, frequent IDH may well aggravate chronic fluid overload due to saline infusions and failure to achieve the prescribed post-dialysis target weight, thereby necessitating a higher ultrafiltration rate during the subsequent treatment and further predisposing the patient to IDH. Lastly, IDH has been linked to mortality [7], which is not surprising given the above.

IDH occurs as a consequence of a reduction in blood volume when the ultrafiltration rate outpaces the vascular refilling rate from the interstitial space. Ultrafiltration rates are largely a function of treatment frequency and duration and, as such, are dictated by dialysis practice. Attempts to reduce the rate of interdialytic weight gain, while valuable in principle, are arguably of limited impact. So, when operating within the constraints of current dialysis practice where the risk for IDH is high, being able to detect impending IDH with sufficient lead time to prevent it from manifesting becomes a primary goal in the efforts to improve dialysis therapy and, ultimately, patient outcomes.

At the same time, blood volume is of key importance in the long run, because in fluid overloaded patients, it is arguably the excess blood volume that mediates most of the cardiovascular damage (vascular stiffening, left ventricular hypertrophy, congestive heart failure), and not the excess interstitial volume.

While IDH takes center stage in most discussions centering around blood volume in dialysis patients, knowledge of absolute blood volume may well have other important ramifications in this population, as we will discuss later in this review. But first, a cursory look at the physiology of the circulatory system is warranted.

\section{The Basics of Acute Circulatory Regulation}

Circulatory regulation is immensely complex, and this review can only touch on some of the cornerstones. The reader is referred to a comprehensive textbook of physiol- ogy for a full discussion of all the involved facets. The purpose of blood circulation, broadly speaking, is to supply oxygen and nutrients to the tissues, to remove carbon dioxide and other metabolic waste products from the tissues, and to distribute humoral transmitters throughout the body. The blood flow through the various tissues is controlled by the respective metabolic needs of these tissues, but in order for there to be any blood flow (i.e. circulation) at all, both arterial blood pressure and cardiac output are necessary. Arterial blood pressure itself is a function of cardiac output and total peripheral resistance of the vasculature. Hence, there are three interconnected parameters that characterize the state of the circulatory system: cardiac output, arterial blood pressure and total peripheral resistance, and the relationship between them is the following:

$$
\text { Cardiac output }=\frac{\text { mean arterial pressure }}{\text { total peripheral resistance }}
$$

Blood flow rate through the tissues is largely regulated locally on the tissue level according to each tissue's metabolic needs at any given time, and is mediated via constriction or dilation of the arterioles, metarterioles and precapillary sphincters. The autonomic nervous system also modulates vasoconstriction/vasodilation and contributes to the regulation of tissue blood flow. In other words, these changes in tissue blood flow are effected via changes in total peripheral resistance - vasoconstriction goes along with an increase in total peripheral resistance, while vasodilation lowers total peripheral resistance. As can be seen in equation 1 , for any given cardiac output, a change in total peripheral resistance will cause an inverse change in arterial pressure.

These changes in arterial pressure are sensed at various levels and trigger counter-regulatory mechanisms aimed at restoring arterial pressure back to normal. The most important one on the arterial side is the baroreceptor reflex system. Baroreceptors in large arteries (aortic arc, carotid sinus) sense arterial pressure changes and elicit autonomic nervous system responses that act on three levels to restore blood pressure. For a drop in arterial pressure, these are: (1) arteriolar constriction - this increases total peripheral resistance and raises blood pressure, (2) constriction of large vessels (primarily veins), thereby displacing blood into the central circulation, which leads to increased cardiac contractility, increased ejection fraction and increased cardiac output via the Frank-Starling mechanism, and (3) direct autonomic regulation of the heart, causing a rise in heart rate, increased contractile force and shortening of the atrio-ven- 
tricular conduction time. In the event of a blood pressure rise, the respective opposite effects occur. Another system to detect changes related to arterial pressure, although this is not its primary function, is the chemoreceptor reflex system: chemosensitive cells in the carotid bifurcation and next to the aorta sense decreases in oxygen supply as well as buildup of hydrogen ions and carbon dioxide that go along with reduced blood flow as a consequence of a drop in arterial pressure (or the reverse in the case of a blood pressure rise). These chemoreceptors then cause a reflective response of the autonomic nervous system.

Changes in blood volume initially affect primarily the venous side of the circulation: they alter the cardiac filling pressure and then secondarily impact cardiac output, which ultimately affects arterial blood pressure. The change in cardiac output also alters total peripheral resistance via autoregulation of the vasculature, which contributes to the change in arterial pressure. But in addition to the baroreceptors on the arterial side, there are similar stretch receptors upstream in the atria and the pulmonary arteries that specifically detect changes in the lowpressure circulation (as brought about by changes in blood volume). These elicit autonomic nervous system responses (as outlined above) and greatly help in blunting arterial pressure changes in response to changes in blood volume. Atrial stretch further increases the heart rate, partially via the direct effect of sinus node stretch but mostly via the Bainbridge reflex, which leads to autonomic nervous system effects on the heart in the form of positive inotropy and positive chronotropy.

The responses discussed above are acute in nature and, as such, are the first line of defense in situations with acute changes in blood volume. Medium- and long-term responses to changes in arterial pressure or blood volume, many of which involve the kidneys, will not be discussed here.

\section{Factors Affecting Blood Volume}

There are in principle two broad categories of events that can alter the blood volume: absolute changes in body fluid volume and changes in body fluid distribution. Changes in fluid distribution between the interstitial space and the vascular space can occur as a result of changes in capillary hydrostatic pressure, plasma oncotic pressure (e.g. hypoalbuminemia), capillary permeability or impaired lymphatic drainage. Absolute changes in fluid volume are self-explanatory. Examples in hemodialysis patients include fluid intake that exceeds urine output, diarrhea, vomiting, blood loss, intravascular infusions of saline or blood, intradialytic ultrafiltration, and others. Since the intravascular water is in equilibrium with the interstitial compartment, any change in extracellular fluid volume will, within a certain range, affect both the interstitial volume and the blood volume. When kidney function is normal, even a wide range of fluid intakes can be handled with only mild changes in extracellular volume (ECV), including blood volume. In the typical dialysis patient, however, fluid intake between dialyses expands both the overall ECV and the blood volume. Over a certain range, the relationship between ECV and blood volume is linear, but with large ECV expansions, blood volume eventually falls behind, and almost all of the additional fluid distributes into the interstitial space. This happens because blood volume expansion ultimately leads to marked rises in intravascular filling pressure, while the interstitial tissue is very compliant and, therefore, acts as a spillway basin for the vasculature. The clinical manifestation of this is edema. Lastly, certain co-morbidities can go along with altered blood volume and, subsequently, ECV (e.g. congestive heart failure and liver disease).

The Special Case of Ultrafiltration during Hemodialysis As noted in the Introduction, the special case of ultrafiltration during dialysis is of particular importance because of its associated sequelae. Without ultrafiltration, IDH is a very rare occurrence, suggesting that ultrafiltration is the prime driver of IDH. The strain put on the cardiovascular system by intradialytic ultrafiltration is immense. It is not uncommon to see filtration volumes over a 3.5- to 4-hour dialysis treatment in the range of 2-3 liters. In a patient with a blood volume of 4.5 liters and a hematocrit of $35 \%$, this amounts to a filtration volume of somewhere from two thirds up to the entire plasma volume. The effective net drop in blood volume in the face of ongoing ultrafiltration will depend on plasma refilling rate, i.e. the rate of fluid transfer from the interstitial compartment into the vasculature, which itself varies throughout the dialysis treatment and depends on the Starling forces (hydrostatic and oncotic pressure differences across the capillary wall). These are a function of various things, such as arteriolar tone, venous tone, changes in plasma solute concentrations (acid-base, electrolytes, proteins), capillary permeability (protein retention), and also the overall fluid volume state of the patient. Plasma refilling rates are on average higher in patients with higher degrees of fluid overload, and lower in patients who are closer to their 'dry weight' [8]. As a re- 
sult, the net change in blood volume can vary widely between patients (and even within a given patient over time) for similar ultrafiltration rates.

The normal acute response to such an ultrafiltrationinduced drop in blood volume starts with the cardiopulmonary stretch receptors picking up the volume reduction and eliciting an autonomic nervous system response resulting in increased arteriolar tone (direct increase in afterload to maintain arterial blood pressure), increased contraction of the venous capacitance vessels (increase in central blood volume, cardiac filling pressure and cardiac output to maintain arterial pressure), and direct cardiac stimulation (increase in heart rate and contractility and, consequently, cardiac output, to maintain arterial pressure). The arteriolar constriction also lowers the capillary pressure and permits an increased fluid flux from the interstitial space into the vasculature to reconstitute the blood volume. If these counter-regulatory mechanisms are not sufficient to prevent a 'spillover' into the arterial side of the systemic circulation, baroreceptors there pick up the drop in arterial pressure and further enhance the autonomic system response as outlined above.

In dialysis patients, however, several factors complicate this normal response: congestive heart failure and diastolic dysfunction are common among hemodialysis patients. These can limit cardiac reserve and make the maintenance of cardiac output and arterial pressure particularly susceptible to drops in cardiac filling pressures as occur as a result of blood volume reduction. Autonomic neuropathy (such as uremic or, particularly, diabetic in nature) is often present as well, although the degree of its contribution to IDH is not as clear-cut [9-11]. Heat accumulation in the patient as a result of the hemodialysis procedure can lead to vasodilation of thermoregulatory cutaneous vessels, which can counter the vasoconstrictive autonomic response to a drop in blood volume and predispose the patient to IDH $[12,13]$. Further, plasma electrolyte and acid-base changes (potassium, calcium, bicarbonate) induced by the dialysis procedure may impair cardiac contractility or, in the case of calcium, affect peripheral vascular tone directly. As far as vascular tone goes, both the arterial and venous systems are of importance, and they are interdependent. During an IDH episode, local tissue hypoperfusion may cause arteriolar dilation (via tissue autoregulation). This not only lowers arterial pressure directly, but it also allows greater transmission of the arterial pressure into the venous system, causing the veins to distend and increase their capacitance (called De Jager-Krogh phenomenon), which in turn can lead to marked reductions in cardiac filling pressure and cardiac output and further reduction in arterial pressure [3]. This may impair tissue perfusion even further and lead into a vicious cycle. Another phenomenon to be aware of is the Bezold-Jarisch reflex, which likely explains the paradoxical withdrawal of sympathetic activity sometimes observed in hemodialysis patients before the onset of hypotensive episodes [14, 15]: as central blood volume and cardiac blood return decrease, vigorous contractions of the poorly filled cardiac ventricles are sensed by ventricular receptors and, in a cardioprotective effort, this information is transmitted to the vasomotor center in the brainstem and translated into a strong parasympathetic activation and sympathetic withdrawal, leading to paradoxical bradycardia and vasodilation and, consequently, an exacerbation of arterial hypotension. Left ventricular hypertrophy with relative myocardial wall ischemia may predispose patients to activation of the Bezold-Jarisch reflex [3]. The latter two mechanisms explain why hypotensive episodes in hemodialysis patients may not be (and often are not) preceded by sudden drops in blood volume.

\section{Absolute or Relative Blood Volume Change - Which Is More Relevant?}

As far as maintenance of cardiac output, blood pressure and tissue perfusion are concerned, it is actually central blood volume (i.e. the blood volume in the heart, the pulmonary circulation and the intrathoracic segments of the large arteries and veins) that matters. As total blood volume decreases, counter-regulatory mechanisms can maintain central blood volume up to a point. One might think, therefore, that measurement of central blood volume is of greatest value. However, central blood volume does not necessarily indicate how much the counter-regulatory system is strained at any given point in time. For this, knowledge of the total absolute blood volume would appear to be far more relevant. It stands to reason that the counter-regulatory reserve is substantially more strained with a total blood volume $20 \%$ below normal than with a normal blood volume. Central blood volume, however, may be nearly the same in both situations. So, for the purpose of predicting impending circulatory breakdown, total blood volume arguably carries more information than central blood volume.

Relative blood volume, in the context of intradialytic blood volume measurements, refers to the ratio of the current blood volume to the initial blood volume at the start of the treatment, typically expressed as a percentage. 
Absolute blood volume can easily be converted to relative blood volume. The same is not true in the opposite direction. All else being equal, one would prefer absolute over relative blood volume for this reason alone. But that aside, knowledge of relative blood volume is inferior to knowledge of absolute blood volume in several ways. Pre-dialysis blood volume varies between patients depending on body size, body composition and fluid status. The relative blood volume change during dialysis contains no information regarding the actual absolute post-dialysis blood volume. Two patients with the same drop in relative blood volume can have very different absolute blood volumes at the end of the treatment. Another important limitation of relative blood volume measurements to bear in mind is that, as part of the intradialytic counter-regulation, blood shifts from the micro- to the macrocirculation. Microcirculation refers to vessels $<200 \mu \mathrm{m}$ in diameter. Since the hematocrit is lower in the microcirculation (Fåhræus effect), blood translocation from the micro- to the macrocirculation leads to central hemodilution, which causes the relative blood volume to underestimate the actual drop in absolute blood volume. The same may occur with postural changes immediately before and during dialysis, intradialytic food intake and intradialytic exercise. To complicate matters further, different devices for measuring relative blood volume appear to yield quite different results [16].

As outlined above, absolute blood volume gives an indication of the degree of strain on the counter-regulatory system and is far more likely than central (let alone relative) blood volume to be useful for establishing critical threshold values, possibly stratified by co-morbid conditions like diabetes mellitus or congestive heart failure. Certainly, a 1-liter blood volume drop from 6 to 5 liters can be expected to be less of a concern than a 1-liter drop from 4 to 3 liters, and using relative blood volume automatically reflects this by yielding a larger drop in the latter case, but it is quite conceivable that the actual absolute blood volume level (not the change) still supersedes this information in terms of circulatory relevance.

Goldfarb et al. showed in a cohort of 10 hemodialysis subjects that 8 were hypervolemic and only 2 were normovolemic with respect to their blood volume, as assessed by an isotope dilution method [17]. Relative blood volume does not convey this important information. Further, relative change in blood volume calculated from absolute blood volume measurements pre- and post-dialysis correlated very well with the respective intradialytic relative blood volume measurement using the Crit Line-III monitor (probably indicating that intradialytic changes in $\mathrm{F}$ cell ratio, the ratio between whole-body hematocrit and peripheral hematocrit, are somewhat similar in magnitude in many cases). Further studies employing absolute blood volume measurements are required to characterize the relationships between absolute blood volume and intradialytic complications, and to demonstrate the proposed superiority of absolute blood volume measurements for this purpose.

\section{How to Measure Absolute Blood Volume}

In vivo measurement of blood volume in humans using tracer dilution has been around for about a hundred years [18], and its application using radioisotope tracers is considered the reference method today. It is based on the principle that injection of a defined concentration or activity of a tracer, followed by measurement of its concentration or activity in a subsequently drawn blood sample (after allowing for complete mixing in the bloodstream), allows for calculation of its distribution volume. Despite the obvious usefulness of having information on absolute blood volume in hemodialysis patients, these measurements are not typically performed. The reasons for this are readily apparent: ideally, blood volume measurements should be non-invasive, inexpensive, reliable and quick, and they should further present no disruption to the operational flow of the dialysis clinic and lend themselves to routine application (which precludes the application of radioactivity). The standard blood volume measurement methods available today fail on most of these counts. What is available, as mentioned above, are devices for measuring relative blood volume during hemodialysis. Many nephrologists are under the misapprehension that this technology can only be used for relative blood volume measurements, but this is in fact not the case. In 1987, Schallenberg et al. [19] already proposed a mathematical method to derive absolute blood volume from measurements of relative blood volume around an abrupt change in ultrafiltration rate, and others have subsequently applied and aimed to validate the concept [20, 21]. In brief, the method can be summarized as follows:

If we assume that ultrafiltration and capillary refilling are the only factors that change the blood volume during hemodialysis, then we get the following relationship:

$$
\frac{d}{d t} B V(t)=Q_{r}(t)-Q_{u}(t), \quad 0 \leq t \leq T
$$

with $T$ denoting the total duration of the hemodialysis treatment, $B V$ denoting the blood volume, $Q_{r}$ being the 
capillary refilling rate, $Q_{u}$ being the ultrafiltration rate, and $t$ denoting a time point during the dialysis treatment.

Hemoglobin concentration is defined as

$$
H g b(t)=\frac{H g b_{\text {mass }}(t)}{B V(t)}
$$

with $\mathrm{Hgb}_{\text {mass }}$ denoting the intravascular mass of hemoglobin.

In the above equations, $H g b_{\text {mass }}, B V$, and $Q_{r}$ are unknown.

If we choose $t_{0} \in[0, T]$ and look at an interval $\left[t_{0}-a, t_{0}\right.$ + a], where $a$ is sufficiently small, we can assume that $H g b_{\text {mass }}$ remains constant over this interval, and from equations 2 and 3 we get

$$
H g b_{\text {mass }}\left(t_{0}\right) \frac{d}{d t}\left(\frac{1}{H g b(t)}\right)=Q_{r}(t)-Q_{u}(t), \quad t \in\left[t_{0}-a, t_{0}+a\right]
$$

which can be rearranged to

$$
Q_{r}(t)=Q_{u}(t)+H g b_{\text {mass }}\left(t_{0}\right) \frac{d}{d t}\left(\frac{1}{H g b(t)}\right), \quad t \in\left[t_{0}-a, t_{0}+a\right]
$$

We now introduce a jump in the ultrafiltration rate at time point $t_{0}$ of size $q_{0}$, i.e.:

$$
q_{0}=Q_{u}\left(t_{0}+0\right)-Q_{u}\left(t_{0}-0\right)
$$

Since the capillary refill rate $Q_{r}$ is continuous at $t_{0}$, we get from equation 5

$$
\begin{aligned}
0 & =Q_{r}\left(t_{0}+0\right)-Q_{r}\left(t_{0}-0\right) \\
& =q_{0}+H g b_{\text {mass }}\left(t_{0}\right)\left(\left.\frac{d}{d t}\left(\frac{1}{H g b(t)}\right)\right|_{t=t_{0}+0}-\left.\frac{d}{d t}\left(\frac{1}{H g b(t)}\right)\right|_{t=t_{0}-0}\right)
\end{aligned}
$$

Now, assume we have a device, such as a modified CritLine III monitor, that can provide high-frequency, realtime hemoglobin concentration data. From these data, we can derive the last two terms in equation 7 , i.e. the right-sided and left-sided differentials of 1 /hemoglobin concentration around the time point $t_{0}$ at which the ultrafiltration rate was changed. These are the slopes of the $1 / \mathrm{Hgb}$ time course data just before and after $t_{0}$, which can be obtained by regression from the raw data. At the same time, $Q_{r}\left(t_{0}+0\right)-Q_{r}\left(t_{0}-0\right)$ is equal to zero, as explained above, and the ultrafiltration rate jump $q_{0}$ is known because we have control over the ultrafiltration rate administered by the dialysis machine. This means that the only unknown quantity in equation 7 is the hemoglobin mass at the time when the ultrafiltration jump occurred, $H g b_{\text {mass }}\left(t_{0}\right)$, which we can thereby solve for. Knowing both $H g b_{\text {mass }}\left(t_{0}\right)$ and $H g b\left(t_{0}\right)$, we can refer to equation 3 to calculate the absolute blood volume at time point $t_{0}$. The theoretical and practical details of this method get more nuanced, but the above is the general principle behind it.

At Renal Research Institute, we are currently evaluating the application of this concept with a modified CritLine III monitor capable of providing a very high sampling frequency. The Crit-Line III monitor (Fresenius Medical Care, Waltham, Mass., USA) is a device for noninvasive real-time measurements of hematocrit, relative blood volume and oxygen saturation during hemodialysis. The Crit-Line-derived blood volume estimates will be validated against reference blood volume measurements obtained by tracer dilution using the Daxor BVA-100 analyzer (Daxor Corp., New York, N.Y., USA). If successful, this would allow for seamless integration of routine, noninvasive assessment of absolute blood volume into the dialysis treatment without any disruption to operations in the clinic.

The results of an initial test run using the modified Crit-Line III monitor and the described method for estimation of absolute blood volume are shown in figures 1 and 2. A dialysis treatment was started with an ultrafiltration rate of $60 \mathrm{ml} / \mathrm{h}$. Three minutes after a stable CritLine signal had been obtained, the ultrafiltration rate was increased to $1,050 \mathrm{ml} / \mathrm{h}$, i.e. an ultrafiltration jump of $+990 \mathrm{ml} / \mathrm{h}$ was introduced, which allowed calculation of hemoglobin mass and absolute blood volume at the time of this initial ultrafiltration jump. Absolute blood volume during the remainder of the treatment was then calculated as hemoglobin mass divided by hemoglobin concentration (measured by Crit-Line). Figure 1 shows the time course of absolute blood volume over the entire dialysis treatment. The upward spike at minute 144 is the result of measurement artifacts and has no physiological correlate. Knowledge of absolute blood volume and ultrafiltration rate at any given time during the treatment permits calculation of the cumulative capillary refill over the course of the dialysis session, which is depicted in figure 2. The ultimate goal is to implement and automate this methodology, so that these data can be provided to the healthcare team at the bedside and in real-time during the dialysis treatment, which we are convinced will be significantly more valuable than the provision of relative blood volume data.

One concern with this approach is that the method is based on a systemic hemoglobin measurement, which has to be converted to a whole-body hemoglobin concentration in order to obtain an accurate blood volume estimate. As outlined earlier in this review, hematocrit and hemoglobin concentration are not constant throughout the vasculature. Rather, they are lower in the micro- 
Fig. 1. Time course of absolute blood volume during a hemodialysis treatment, determined from high-frequency hemoglobin readings around a jump in ultrafiltration rate at the beginning of dialysis. Data were sampled using a modified Crit-Line III monitor

Fig. 2. Time course of cumulative capillary refill during a hemodialysis treatment, calculated from absolute blood volume and Crit-Line monitor-derived hemoglobin concentration measurements. At any time point, the sum of the change in blood volume (calculated as starting blood volume minus current blood volume) and the cumulative capillary refill volume yields the cumulative ultrafiltration volume up to that point. In the depicted case, the total ultrafiltration volume at the end of dialysis, e.g., is 3.1 liters (blood volume reduction of 1.3 liters plus cumulative refill volume of 1.8 liters).
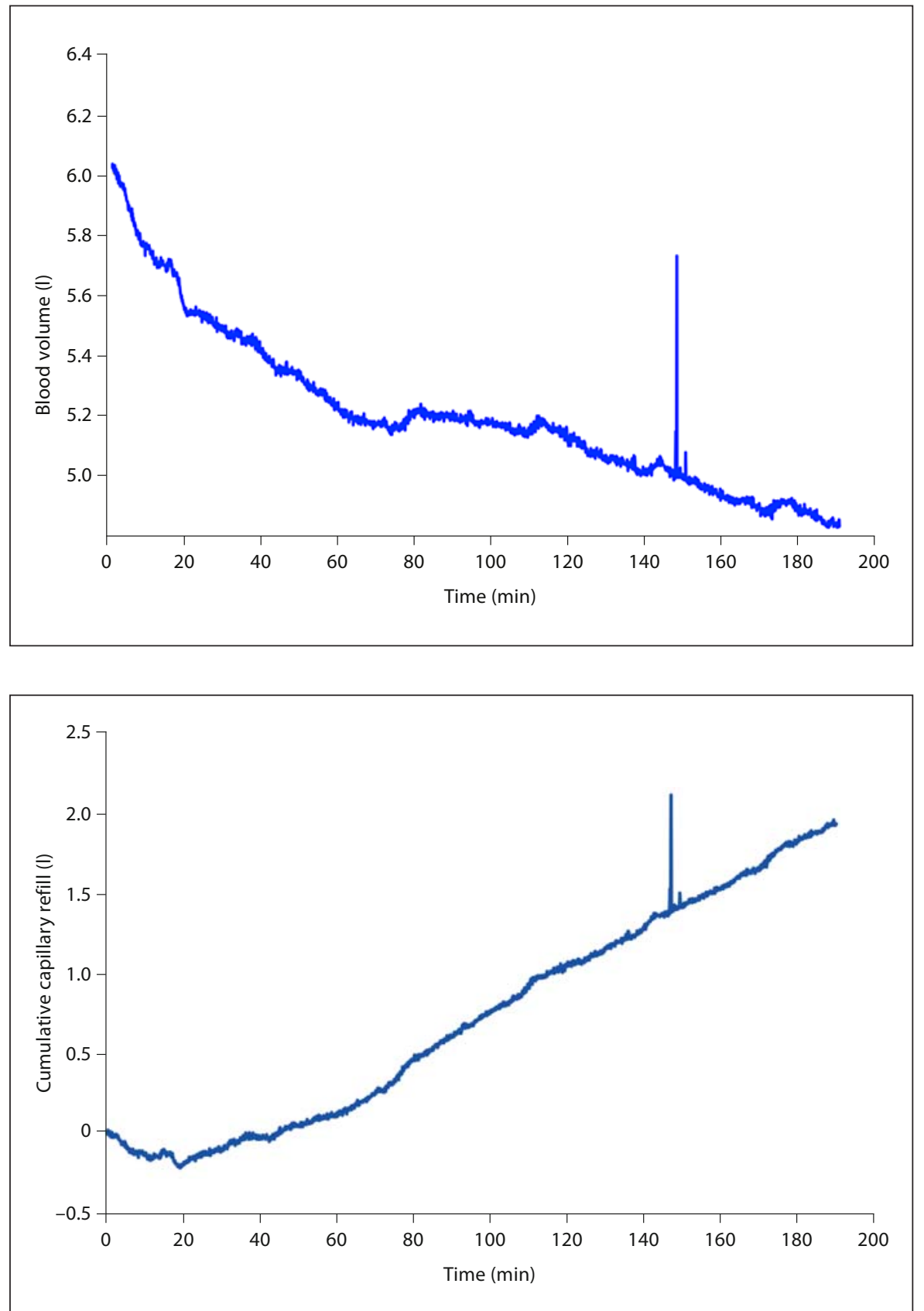

than in the macrocirculation. The ratio of whole-body hematocrit (the average hematocrit of the entire blood in the vasculature) to the systemic hematocrit, also called the F cell ratio, is about 0.91 on average. Using this average ratio, whole-body hematocrit or hemoglobin concentration can be calculated from the systemic values, but this may not reflect the true $\mathrm{F}$ cell ratio in a given patient. Furthermore, it can be expected that ongoing ultrafiltration during hemodialysis may prompt signifi- cant changes in the blood volume distribution between the micro- and macrovasculature, thereby changing the F cell ratio during the course of the treatment. Ultrafiltration during dialysis is in some respects comparable to acute bleeding, and a shift of blood volume from the micro- to the macrocirculation has been documented in rabbits in response to blood loss [22]. The extent of such intradialytic changes in the F cell ratio deserves further study. Ultimately, an automated real-time method for 
assessing the fluid distribution between micro- and macrocirculation would be desirable to eliminate this uncertainty.

\section{Absolute Blood Volume: Implications beyond Intradialytic Hypotension}

IDH certainly takes center stage in most discussions around blood volume, but two other important areas deserve mention in this context as well.

\section{Anemia Management}

Blood volume remains relatively stable in the face of changes in circulating hemoglobin mass, i.e. as the number of red blood cells increases (e.g. in response to erythropoietin therapy), plasma volume shrinks to maintain a stable blood volume. On the other hand, changes in blood volume do affect hemoglobin levels [23]. In other words, knowledge of the absolute blood volume is relevant for assessment of the size of the circulating hemoglobin pool and can help distinguish between patients with too little hemoglobin in circulation and those with adequate hemoglobin mass but suffering from hemodilution. In the future, an advanced application of absolute blood volume will likely come into play as new mathematical models for anemia management find their way into clinical practice. The model recently published by Fuertinger et al. [24], for example, shows remarkable fidelity in preclinical studies for the prediction of red cell kinetics, and knowledge of absolute blood volume is an integral part of such models in order to convert circulating numbers and masses to concentrations.

\section{The Quest for 'Dry Weight' - Time for a Paradigm Shift?}

Quite aside from its importance for acute hemodynamic stability, absolute blood volume can be argued to have important implications for how we approach the question of dry weight attainment in the broader context. Defining dry weight is a challenge in and of itself, and the trend goes toward objective quantification of fluid status by bioelectrical impedance analysis. Knowledge of a patient's whole-body fluid status and how it compares to individuals without kidney disease is valuable, but normalizing ECV is not the be all end all of 'dry weight' attainment. It has to be borne in mind that it is not primarily the interstitial fluid that causes hypertension, left ventricular hypertrophy, congestive heart failure and other cardiovascular sequelae of fluid overload - it is the ex- pansion of intravascular volume that mediates this damage. Likewise, it is not reduced interstitial volume but intravascular underfilling that compromises adequate circulation and causes hypoxidotic end-organ damage. Gradually reducing post-dialysis weight towards a welldefined normal range (and objectively monitoring this process) is worthwhile, but we propose that absolute blood volume should be the parameter that defines where this process ends. For a given patient, with a certain physiologic/pathophysiologic situation and a certain treatment regimen (primarily with respect to dialysis duration and frequency), absolute blood volume will dictate how low the post-dialysis weight can be reduced without causing intravascular volume depletion and compromising tissue perfusion. And for a hypoalbuminemic patient, for example, it is conceivable that some degree of interstitial fluid overload is required to maintain an adequate intravascular volume. Normalizing the overall extracellular fluid at the expense of the intravascular volume is not indicated in such a case.

Will such an approach based on absolute blood volume on average lead to higher time-averaged whole-body (and even intravascular) fluid content? Possibly. Perhaps likely. But this is a direct reflection of the current paradigm that says that intravascular volume depletion during dialysis is acceptable and necessary to maximize the mobilization of interstitial fluid. There is compelling evidence that such intravascular underfilling causes profound circulatory stress, which in itself is enough to cause cumulative damage to the heart [6]. But the detrimental effects of intradialytic blood volume depletion extend to various other critical organs, such as the brain, the gut and the kidneys, causing leukoaraiosis, inflammation via endotoxin translocation through the intestinal wall, and accelerated loss of residual kidney function [6]. Of note, some of these negative effects have been shown to occur during ultrafiltration even without overt hypotensive episodes (e.g. myocardial stunning in the presence of relative hypotension, and mesenteric ischemia due to splanchnic hypovolemia) - in other words, the counter-regulatory mechanisms to maintain blood pressure are sufficient in certain cases to mediate end-organ damage. Clearly, it is not sufficient to state the goal as normalizing the timeaveraged blood volume (let alone the time-averaged ECV), because this goal would sanction severe intravascular hypovolemia during dialysis. The goal must be to maximize the time that a patient's blood volume is in the normal range. The notion that intradialytic blood volume depletion is acceptable to reduce the time-averaged fluid load is outdated and harmful. In our opinion, post- 
dialysis weight should be gradually reduced toward an objectively defined (and measurable) target (a discussion of which is beyond the scope of this article), but the path toward this target should be guided and informed by absolute blood volume determinations, and these should also dictate at what body weight this path ends. At that point, it should be considered whether treatment regimen modifications (especially with respect to treatment duration/frequency) are possible and feasible to allow further improvements in fluid volume status.

\section{Conclusion and Outlook}

Intradialytic changes in blood volume mediate cardiovascular complications, both acute (during dialysis) as well as long-term. Knowledge of intradialytic absolute blood volume (assessed on a routine basis) is likely to significantly improve dialysis care in the areas of acute, intradialytic complications (IDH, cramps) as well as medium- and long-term outcomes (preservation of residual renal function, cardiovascular health, cerebral function).
Technology is improving, and methods for routine assessment of absolute blood volume are on the horizon. With such technology routinely available, the goal of maximizing the time that a patient's blood volume is in the normal range could finally be effectively pursued. And if, within the constraints of current dialysis practice, the avoidance of intravascular hypovolemia cannot be accomplished without an unacceptable rise in timeaveraged blood volume, then the question should not be whether blood volume excess or intradialytic blood volume depletion is the lesser evil that we should favor. While academically interesting, it is a false dichotomy if we are serious about the well-being of our patients. Instead, the focus should be on changing the framework to allow delivery of hemodialysis in a way that minimizes both of these detrimental conditions.

\section{Acknowledgement}

The authors acknowledge the critical review of the manuscript by Dr. Nathan W. Levin, Renal Research Institute, N.Y., USA.

\section{References}

1 US Renal Data System, USRDS 2012 Annual Data Report: Atlas of End-Stage Renal Disease in the United States. Bethesda, National Institutes of Health, National Institute of Diabetes and Digestive and Kidney Diseases, 2012, p 225.

12 Daugirdas JT: Preventing and managing hypotension. Semin Dial 1994;7:276-283.

-3 Daugirdas JT: Pathophysiology of dialysis hypotension: an update. Am J Kidney Dis 2001;38(suppl 4):S11- S17.

4 Thijssen S, et al: The evils of intradialytic sodium loading. Contrib Nephrol. Basel, Karger, 2011, vol 171, pp 84-91.

5 Eldehni MT, McIntyre CW: Are there neurological consequences of recurrent intradialytic hypotension? Semin Dial 2012;25:253256.

6 McIntyre CW: Recurrent circulatory stress: the dark side of dialysis. Semin Dial 2010;23: 449-451.

7 Shoji T, et al: Hemodialysis-associated hypotension as an independent risk factor for two-year mortality in hemodialysis patients. Kidney Int 2004;66:1212-1220.

8 Wizemann V, et al: Influence of hydration state on plasma volume changes during ultrafiltration. Artif Organs 1995;19:416-419.

9 Ligtenberg G: Regulation of blood pressure in chronic renal failure: determinants of hy- pertension and dialysis-related hypotension. Neth J Med 1999;55:13-18.

10 Raine AE: The susceptible patient. Nephrol Dial Transplant 1996;11(suppl 2):6-10.

-11 Chang MH, Chou KJ: The role of autonomic neuropathy in the genesis of intradialytic hypotension. Am J Nephrol 2001;21:357-361.

12 Schneditz D, Levin NW: Keep your temper: how to avoid heat accumulation in haemodialysis. Nephrol Dial Transplant 2001;16:7-9.

13 Van der Sande FM, et al: Energy transfer is the single most important factor for the dif ference in vascular response between isolated ultrafiltration and hemodialysis. J Am Soc Nephrol 2000;11:1512-1517.

14 Pelosi G, et al:Impaired sympathetic response before intradialytic hypotension: a study based on spectral analysis of heart rate and pressure variability. Clin Sci 1999;96:23-31.

15 Converse RL Jr, et al: Paradoxical withdrawal of reflex vasoconstriction as a cause of hemodialysis-induced hypotension. J Clin Invest 1992;90:1657-1665.

16 Dasselaar JJ, et al: Relative blood volume measurements during hemodialysis: comparisons between three noninvasive devices. Hemodial Int 2007;11:448-455.

17 Park JK, Mattoo A, Modersitzki F, Goldfarb DS: Radioistope blood volume (BV) measurement in conjuction with Crit-Line
(CLM-III) in hemodialysis (HD) patients [Abstract]. J Am Soc Nephrol 2012;23: 257A.

18 Keith NMR, Rowntree LG, Geraghty JT: A method for the determination of plasma and blood volume. Arch Intern Med 1915;16: 547-576.

19 Schallenberg U, Stiller S, Mann H: A new method of continuous haemoglobinometric measurement of blood volume during haemodialysis. Life Support Syst 1987;5:293-305.

20 Leypoldt JK, et al: Determination of circulating blood volume by continuously monitoring hematocrit during hemodialysis. J Am Soc Nephrol 1995;6:214-219.

21 Johnson DW, et al: Non-invasive, optical measurement of absolute blood volume in hemodialysis patients. Kidney Int 1996;49: 255-260.

22 LaForte AJ, et al: Fluid restitution and shift of blood volume in anesthetized rabbits subject to cyclic hemorrhage. Am J Physiol 1992; 262:H190-H199.

23 Bellizzi V, et al: Influence of the cyclic variation of hydration status on hemoglobin levels in hemodialysis patients. Am J Kidney Dis 2002;40:549-555.

24 Fuertinger DH, et al: A model of erythropoiesis in adults with sufficient iron availability. J Math Biol 2012, E-pub ahead of print. 